\title{
Pediatric achalasia. Single-center study of interventional treatment
}

\author{
Andrzej Grabowski, Wojciech Korlacki, Michał Pasierbek, Roksana Puttorak, Filip Achtelik, Maciej Ilewicz \\ Department of Children's Developmental Defects Surgery and Traumatology in Zabrze, Medical University of Silesia \\ in Katowice, Poland
}

Gastroenterology Rev 2017; 12 (2): 98-104

DOI: https://doi.org/10.5114/pg.2016.64845

Key words: achalasia, children, pneumatic dilatation, cardiomyotomy.

Address for correspondence: Andrzej Grabowski MD, PhD, Department of Children's Developmental Defects Surgery and Traumatology, Medical University of Silesia, ul. 3 maja 13-15, 41-800 Zabrze, Poland, phone: +48 691751 393, e-mail: agrabowski@szpital.zabrze.pl

\begin{abstract}
Introduction: Esophageal achalasia is a rare entity in children. However, young age is a factor of failure of conservative treatment, emphasizing the role of surgery. In our institution laparoscopic Heller's cardiomyotomy is the chosen procedure for surgical treatment.

Aim: To assess the outcome of surgery for achalasia treatment in children operated on in a single institution.

Material and methods: A retrospective analysis of consecutive patient records from the years 1997 to 2014 was performed. There were 11 patients. Their mean age was 13 years, ranging from 6 to 17. Duration of symptoms was 2 to 36 months, mean 16. All 11 patients were operated on with a laparoscopic approach. Pneumatic dilatation was used both pre- and postoperatively but in no case was sufficient on its own. Collected data included patient demographics, preoperative symptoms and their duration, diagnostic findings and therapeutic means. Surgical procedures, complications and long-term follow-up were analyzed. The follow-up lasted from 1 to 10 years and finished when the patient reached 18 years of age.

Results: Twelve laparoscopic cardiomyotomies were performed with concomitant fundoplications, 10 Toupet and 2 Dor and one redo procedure. There were no deaths. Two perforations were repaired promptly. The success rate was $82 \%$, though with subsequent dilatations. One failure was due to serious progression of the disease.
\end{abstract}

Conclusions: In our opinion, laparoscopic Heller's myotomy is the procedure of choice for treating achalasia in children. Endoscopic balloon dilatation may be used as a complementary treatment, especially as a primary redo procedure.

\section{Introduction}

Esophageal achalasia is an obstructive disorder of the esophagus with aperistalsis and failure of the lower esophageal sphincter (LES) to relax in response to swallowing. Progression of the disease leads to dilatation of the esophageal body and consequent loss of its peristalsis. As the primary cause is degeneration of inhibitory myenteric neurons, cure is not achievable.

Therapy aims to release the LES pressure, widen the esophageal lumen and improve its outflow. Well-established methods include endoscopic balloon dilatation, Heller's myotomy and botulinum injections [1]. Recent reports also mention per oral endoscopic myotomy (POEM) [2] and esophageal stenting [3].

The overall incidence of achalasia is $1.63: 100000$, fewer than $5 \%$ of whom are children $[4,5]$. However, young age at presentation is an independent predictive factor of failure of conservative treatment [6].

\section{Aim}

We wanted to assess the outcome of laparoscopic Heller's cardiomyotomy for achalasia treatment in children operated on in a single institution.

\section{Material and methods}

A retrospective analysis of consecutive patient records with radiologically proven and symptomatic achalasia was performed from the years 1997 to 2014. There were 11 patients who had laparoscopic cardiomyotomy in 12 operations. Collected data included patient demographics, pre-operative symptoms and their duration, diagnostic findings and therapeutic means. Surgical procedures, complications and long-term follow-up were analyzed.

\section{Results}

There were 11 patients in the study. Their mean and median age on the day of operation was 13 years, 


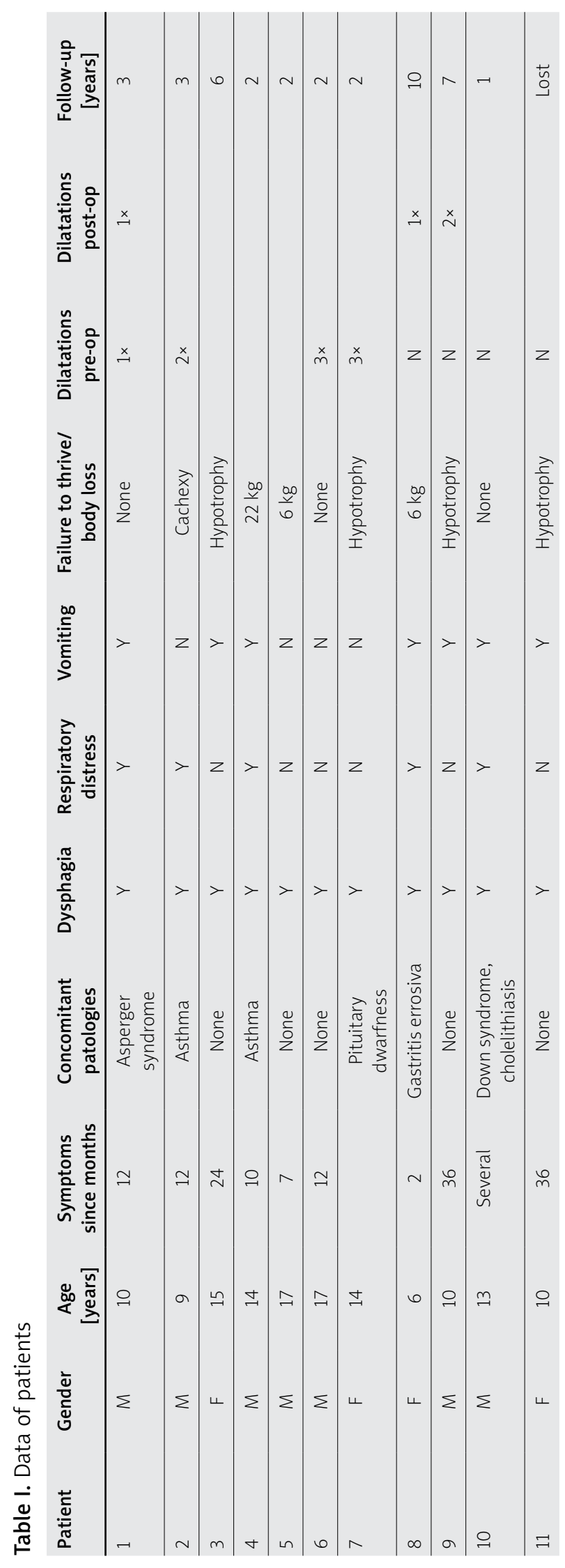

ranging from 6 to 17. Duration of symptoms was 2 to 36 months, median 12 . The symptoms were dysphagia $(100 \%)$, vomiting and regurgitation (90\%), and respiratory distress - recurrent pneumonia or asthma (45\%). Seventy percent failed to thrive or had body mass loss the largest was $22 \mathrm{~kg}$, two were $6 \mathrm{~kg}$, and the rest were outside the range of expected body mass and height. One was diagnosed with pituitary dwarfism prior to the achalasia symptoms (Tables I, II).

There were no hereditary (Allgrove syndrome) or evident contagious causes of disease.

Diagnoses were mostly made from an esophagogram with the typical "bird's beak" appearance in all patients. Moreover, 7 (63\%) patients had manometry with typical loss of coordination in relaxation of LES combined with ineffective peristalsis of the lower portion and - in 1 case - of the whole body of the esophagus. In 5 patients esophagoscopy was conducted, which in 4 showed a dilated esophagus and difficulty in negotiating the instrument through the cardia (Table II, Figures 1, 2).

Table II. Symptoms and diagnostics means conducted

\begin{tabular}{lcc} 
Symptoms & Frequency & Percentage \\
\hline Dysphagia & 11 & 100 \\
\hline Vomiting, regurgitation & 10 & 90 \\
\hline Respiratory distress & 5 & 45 \\
\hline Failure to thrive or body mass lost & 8 & 70 \\
\hline Preoperative diagnostics: & & \\
\hline X-ray & 11 & 100 \\
\hline Manometry & 7 & 63 \\
\hline Esophagoscopy & 4 & 37
\end{tabular}

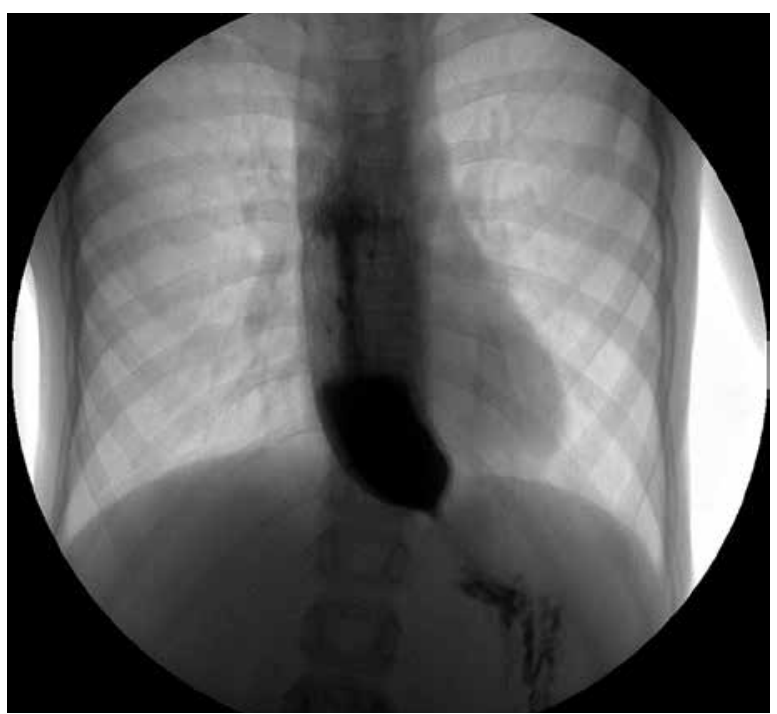

Figure 1. X-ray with the typical "bird's beak" 

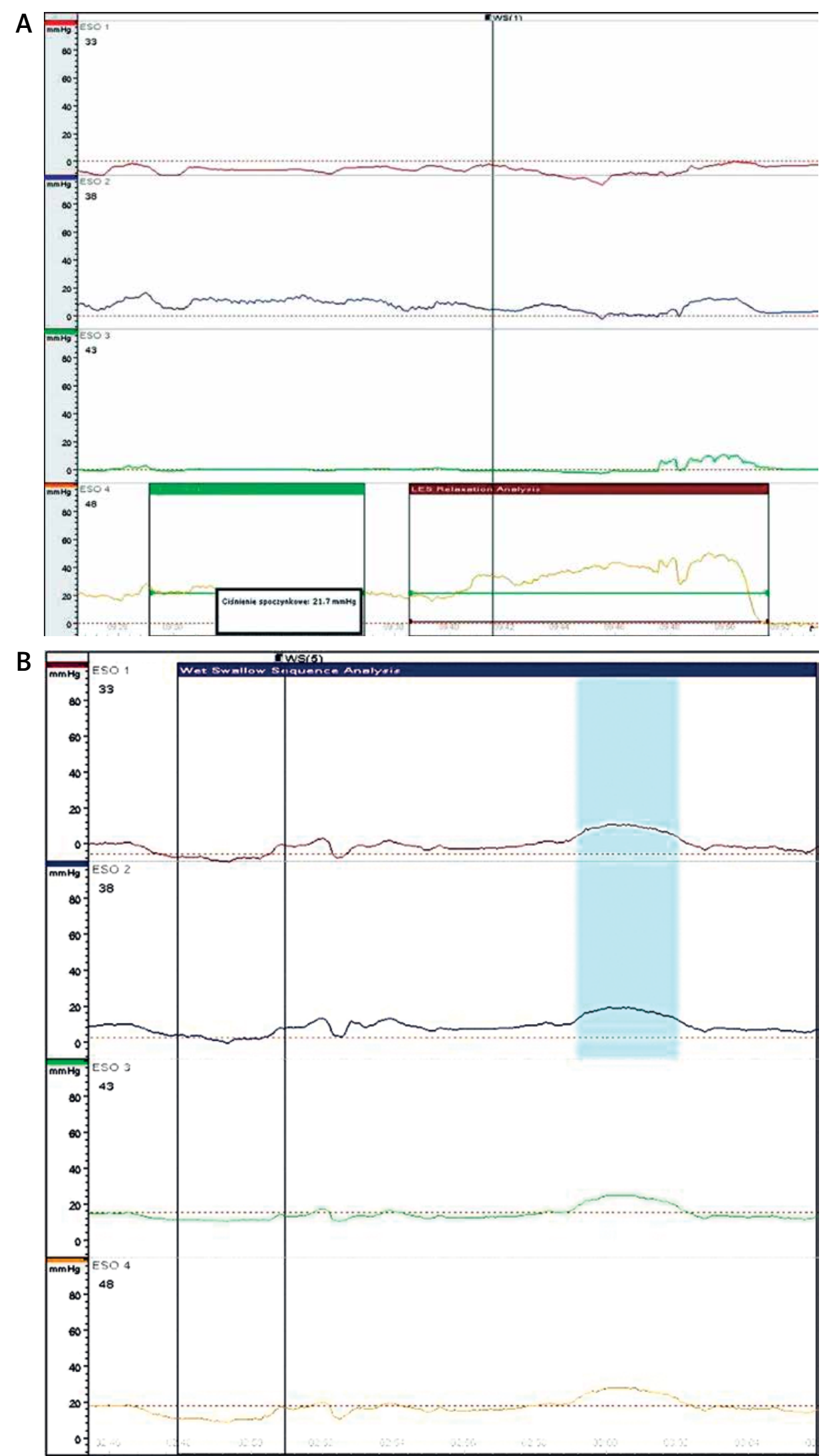

Figure 2 A, B. Manometry. Low peristalsis during the wet swallow. No relaxation at the LES level 
Four patients underwent endoscopic balloon dilatation - two of them three times, one twice, one once. No specific protocol was used due to the small number of patients, and each patient was treated individually. The intervals depended on the symptoms. The decision of the method used (i.e. dilatations or surgery) was taken by caregivers after full information. Despite primary relief, conservative therapy eventually failed in all of them, and they were referred for surgery.

All 11 patients were operated on with a laparoscopic approach by two consultant surgeons.

After wide esophagus mobilization in the hiatal region, the muscular layer in the distal part of the esophagus and cardia was cut over a length of $6-8 \mathrm{~cm}$ depending on patient age. Special attention was paid to cutting the muscle $1 \mathrm{~cm}$ below the gastro-esophageal junction and to cutting all muscular fibers. Scissors or a harmonic knife (SonoSurg, Olympus Medical Systems Corp., Tokyo, Japan and Autosonix AutoSuture, Covidien, Mansfield, USA) were used for this maneuver (Figures 3-5).

In 9 cases concomitant posterior fundoplication according to Toupet was done to improve opening the muscular layer and to prevent future GER symptoms.

Intraoperatively, 2 (16\%) patients had mucosal perforations. The site of mucosal perforation was primarily sutured and, in these cases, Dor anterior fundoplication was performed to protect the mucosa.

There were no deaths, major intraoperative bleeding or conversions. In the immediate postoperative period no complications were observed. The mean hospital stay was 8 days, ranging from 5 to 13 days, significantly longer in patients after perforations due to the delay in oral feeding. The mean postoperative fast was 3 days, range $2-7$ days.

The follow-up lasted from 1 to 10 years, median 2.5 years. One patient was lost to follow-up for unknown reasons. Three are still being followed. After the operation all patients declared relief of symptoms. All of them attained appropriate weight.

In 6 patients the improvement was lasting. Four had recurrence of dysphagia over a period of 2 months to 8 years. Three of them had endoscopic balloon dilatation performed (once or twice); in 2 cases it was curative. One patient refused further treatment, declaring sufficient quality of life. One patient was referred for a redo procedure. Intraoperatively no adverse features were found. The wound was healed, and the Toupet's cuff was intact. Laparoscopic Heller's myotomy was performed again with no intra- or postoperative complications. No major improvement was gained. That patient

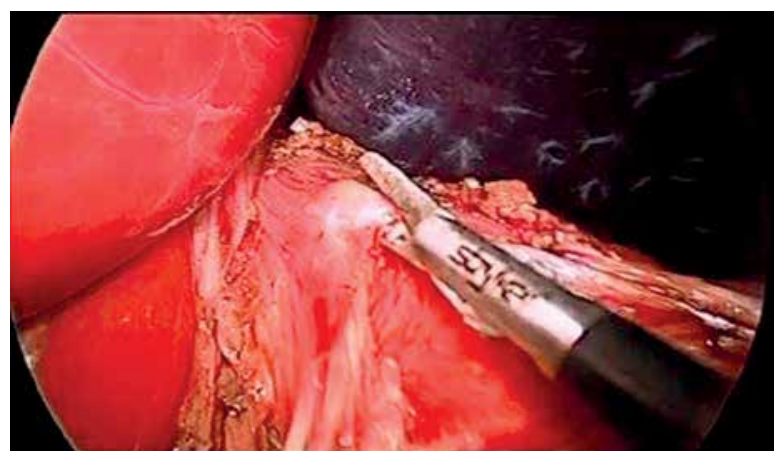

Figure 3. Laparoscopy view. Cutting the muscular layer

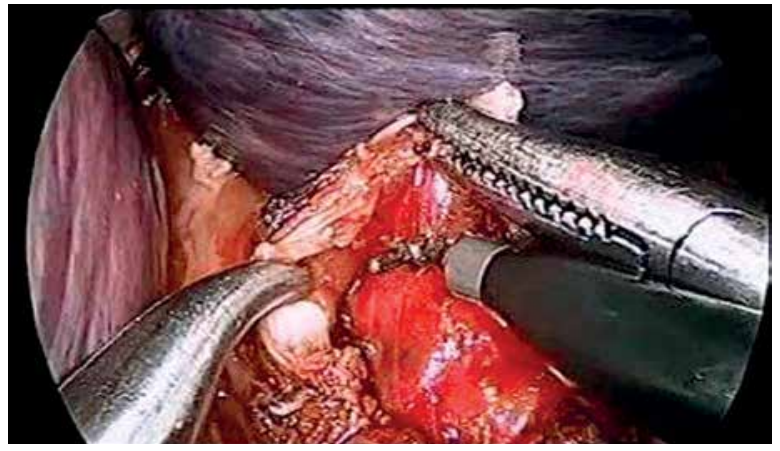

Figure 4. Laparoscopy view. Releasing the mucosa from the muscular layer

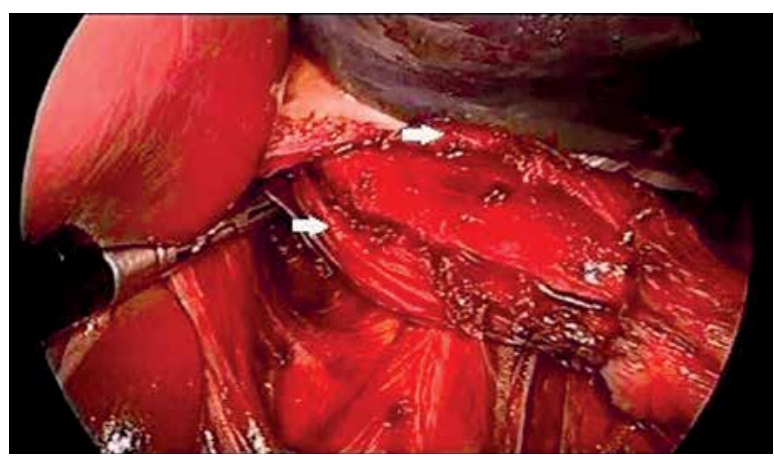

Figure 5. Laparoscopy view. Muscular layer cut - its margins marked with arrows. Bare mucosa seen in the center

was diagnosed in further follow-up via fluoroscopy and manometry with complete aperistalsis of the entire esophageal body, recognized as progress of the disease in 8-year follow-up. No further treatment except antacids was conducted, but the patient states that she manages to swallow solid food.

Thus, 2 patients prospered even with failure of operational treatment but subjectively with adequate quality of life and ability to maintain proper weight.

There were no gastroesophageal reflux related morbidities, though 2 patients are administered oral antacid medication permanently (Table III). 
Table III. Surgical outcomes

\begin{tabular}{|c|c|c|c|}
\hline Procedures & Frequency & Percentage & Observation \\
\hline Baloon dilatation preop. & 4 & 34 & \\
\hline Laparoscopic Heller's myotomy: & 11 & 100 & \\
\hline Toupet's fundoplication & 9 & 81 & \\
\hline Dor fundoplication & 2 & 19 & In case of perforation \\
\hline \multicolumn{4}{|l|}{ Complications: } \\
\hline Conversion & 0 & 0 & \\
\hline Perforation & 2 & 18 & \\
\hline Hemotransfusion & 0 & 0 & \\
\hline ICU stay & 0 & 0 & \\
\hline Mortality & 0 & 0 & \\
\hline Follow up: & 10 & 90 & Range $1-10$ years \\
\hline Initial success: & 6 & 55 & \\
\hline Baloon dilation postop. & 3 & 27 & \\
\hline Reoperation & 1 & 9 & \\
\hline Overall success & 8 & 72 & \\
\hline Failure & 2 & 18 & $\begin{array}{c}\text { One case of complete aperistalsis of the entire } \\
\text { esophageal body }\end{array}$ \\
\hline
\end{tabular}

\section{Discussion}

Achalasia is known to have been treated since the year 1674 with dilatations, but Heller's method was introduced in 1913. Since the pathology is rare and children represent less than $5 \%$ of patients, there is a lack of large group reports and grade 1 or 2 evidence.

The tools for diagnosis are well established: in most children the oral contrast swallow study is diagnostic. Sixty-three percent of our patients had the diagnosis confirmed with esophageal manometry with typical findings [7].

The proper treatment is what is still debatable. Though laparoscopic Heller myotomy seems to be the method of choice, there are still opinions in favor of other methods. Concern is focused on conservative treatment versus operation, with the POEM being a novelty in the field.

Endoscopic balloon dilatation seems to be first choice treatment due to its low morbidity and the ease of the procedure, but the risk of recurrence reaches $100 \%$ during long-term follow-up, and young age at presentation is an independent predictive factor for the need for repeated treatment $[6,8]$.

This does not exclude the method from use in initial treatment or - particularly - in the treatment of postoperative recurrences. In both circumstances the method was used in the group and the latter gave an overall $82 \%$ success rate.

The use of intersphincteric botulinum injection has a high failure rate - in fact all children in a reported series required retreatment [9]. This clearly limits its use as an efficacious treatment modality, given the need for repeated admissions and general anesthesia, and it should be reserved for exceptional cases [10]. Moreover, previous botulinum injections cause the formation of scar tissue around the cardia and distal part of the esophagus, which makes myotomy much more difficult.

According to the literature, only operational treatment via Heller's myotomy modified to a single incision brings lasting relief of symptoms, as other methods need repetition [6]. There are no papers which can give clear recommendations for the method, because of the lack of randomized studies in children and because the reports concern small groups only. The largest pediatric group found, the 44 patients from the GOSH, were all treated with Heller's myotomy despite different approaches to primary intervention [1]. The same was reported by Lee et al. and Mattioli et al. [8, 11]. The meta-analysis done by Schoenberg et al. to reveal whether balloon dilatation is as good as myotomy again demonstrated superior short- and long-term efficacy of the latter [12]. 
The POEM started in children in 2012 with 1 case [13], soon followed by small groups of Familiari et al. and Caldaro et al. [2, 14]. The method seems equally efficient but less invasive in those reports. Future prospective evaluation will need to be conducted to ascertain whether POEM is safe and equally effective as the Heller myotomy in children.

Another controversy concerns the need for concomitant fundoplication. Most authors treat fundoplication as an essential part of the procedure $[11,15$, 16]. A randomized, double-blind clinical trial by Richards et al. of 43 adults proved that the incidence of postoperative gastroesophageal reflux disease (GERD) symptoms was higher in the group without fundoplication [17]. However, a later paper reported more and more patients treated without fundoplication with a satisfactory success rate and without GERD symptoms $[1,18]$ The authors suggested that this is related to the length of the incision, especially its gastric portion. But again, there is no definitive high-grade evidence to indicate one course or the other and, in particular, no reports of adults being treated as children without fundoplications, so we decided to do the fundoplication in most cases so far. We chose Toupet's procedure not only to secure the esophagus from reflux but to keep the myotomy groove wider, which is however a personal conviction not clearly based on literature. In all of our cases we managed to avoid inadequate myotomy, as the only reoperated patient proved to have aperistalsis along the entire esophagus, and thus could not be cured even after a second procedure. The other cases were patients with a perioperative perforation where anterior fundoplication (Dor's) secured the suture line; thus, it is strongly advised.

In our group we had a good outcome in the majority of cases, although 34\% required some form of re-intervention. This is higher than reported in the literature to date $[1,15]$, but after reintervention the success rate reached $82 \%$ symptomless patients, with the rest being adequately treated.

\section{Conclusions}

This study adds to the debate in children concerning whether to operate or treat achalasia conservatively and what the place for endoscopic balloon dilatation is in treatment. In our opinion laparoscopic Heller's myotomy is the procedure of choice for treating achalasia in children. Endoscopic balloon dilatation may be used as a complementary treatment, especially as a primary redo procedure, as the muscle has already been divided and dilatation may widen the myotomy further or disrupt any scar tissue or adhesions that may have formed following the myotomy. This is probably why the efficacy of dilatation after myotomy is higher than before [1, 19].

\section{Conflict of interest}

The authors declare no conflict of interest.

\section{References}

1. Pachl MJ, Rex D, Decoppi P, et al. Paediatric laparoscopic Heller's cardiomyotomy: a single centre series. J. Pediatr Surg 2014; 49: 289-92.

2. Familiari P, Marchese M, Gigante G, et al. Peroral endoscopic myotomy for the treatment of achalasia in children. J Pediatr Gastroenterol Nutr 2013; 57: 794-7.

3. Cheng YS, Ma F, Li YD, et al. Temporary self-expanding metallic stents for achalasia: a prospective study with a long-term follow-up. World J Gastroenterol 2010; 16: 5111-7.

4. Sadowski DC, Ackah F, Jiang B, et al. Achalasia: incidence, prevalence and survival. A population-based study. Neurogastroenterol Motil 2010; 22: e256-61.

5. Singh S, Wakhlu A, Pandey A, et al. Retrospective analysis of paediatric achalasia in India: single centre experience. Afr J Paediatr Surg 2012; 9: 117-21.

6. Alderliesten J, Conchillo JM, Leeuwenburgh I, et al. Predictors for outcome of failure of balloon dilatation in patients with achalasia. Gut 2010; 60: 10-6.

7. Franklin AL, Petrosyan M, Kane TD. Childhood achalasia: a comprehensive review of disease, diagnosis and therapeutic management. World J Gastrointest Endosc 2014; 6: 105-11.

8. Lee CW, Kays DW, Chen MK, et al. Outcomes of treatment of childhood achalasia. J Pediatr Surg 2010; 45: 1173-7.

9. Ip KS, Cameron DJ, Catto-Smith AG, et al. Botulinum toxin for achalasia in children. J Gastroenterol Hepatol 2000; 15: 1100-4.

10. Gutschow CA, Töx U, Leers J, et al. Botox, dilation, or myotomy? Clinical outcome of interventional and surgical therapies for achalasia. Langenbecks Arch Surg 2010; 395: 1093-9.

11. Mattioli G, Esposito C, Pini Prato A, et al. Results of the laparoscopic Heller-Dor procedure for pediatric esophageal achalasia. Surg Endosc 2003; 17: 1650-2.

12. Schoenberg MB, Marx S, Kersten JF, et al. Laparoscopic Heller myotomy versus endoscopic balloon dilatation for the treatment of achalasia: a network meta-analysis. Ann Surg 2013; 258: 943-52.

13. Maselli R, Inoue H, Misawa M, et al. Peroral endoscopic myotomy (POEM) in a 3-year-old girl with severe growth retardation, achalasia, and Down syndrome. Endoscopy 2012; 44 Suppl 2 UCTN: E285-7.

14. Caldaro T, Familiari P, Romeo, et al. Treatment of esophageal achalasia in children: today and tomorrow. J Pediatr Surg 2015; 50: 726-30.

15. Askegard-Giesmann JR, Grams JM, Hanna AM, et al. Minimally invasive Heller's myotomy in children: safe and effective. J Pediatr Surg 2009; 44: 909-11.

16. Grubnik VV, Malinovskiy AV, Grubnik AV. Relationship between subjective and objective data in achalasia patients after laparoscopic Heller-Dor procedure. Wideochirurgia i Inne Techniki Małoinwazyjne 2006; 1: 137-41.

17. Richards WO, Torquati A, Holzman MD, et al. Heller myotomy versus Heller myotomy with Dor fundoplication for achalasia: a prospective randomized double-blind clinical trial. Ann Surg 2004; 240: 405-15. 
18. Corda L, Pacilli M, Clarke S, et al. Laparoscopic oesophageal cardiomyotomy without fundoplication in children with achalasia: a 10-year experience: a retrospective review of the results of laparoscopic oesophageal cardiomyotomy without an anti-reflux procedure in children with achalasia. Surg Endosc 2010; 24: 40-4.

19. Jung C, Michaud L, Mougenot JF, et al. Treatments for pediatric achalasia: Heller myotomy or pneumatic dilatation? Gastroenterol Clin Biol 2010; 34: 202-8.

Received: 14.07 .2015

Accepted: 12.01 .2016 